\title{
Texture et évolution du pH de fromages de type Brie entreposés en atmosphère contrôlée
}

\author{
Claude P. CHAMPAGNE ${ }^{\mathrm{a}}$, Lucie SOULIGNAC ${ }^{\mathrm{b}}$, Michèle MARCOTTE ${ }^{\mathrm{a} *}$, \\ Jean-Pierre INNOCENT ${ }^{b}$ \\ ${ }^{a}$ Centre de recherche et de développement sur les aliments (CRDA), Agriculture et Agroalimentaire \\ Canada, 3600, boulevard Casavant Ouest, Saint-Hyacinthe (Québec) J2S 8E3, Canada \\ b SOREDAB, La Tremblaye, 78125, La Boissière École, France
}

(Reçu le 23 juillet 2001 ; accepté le 4 novembre 2002)

\begin{abstract}
Résumé - Des fromages de type Brie ont été entreposés à $7{ }^{\circ} \mathrm{C}$ en atmosphère contrôlée (AC) en faisant varier la teneur en oxygène $(0,5,10$ ou $25 \%)$, en $\mathrm{CO}_{2}(0,5,10,25$ ou $50 \%)$ et en azote (30 à $70 \%$ ). L'effet de l'AC sur l'évolution du $\mathrm{pH}$, l'activité protéolytique et la texture des fromages ont été étudiés. La consommation d'acide lactique observée en conditions aérobies était corrélée à la hausse du pH. Il n'y avait pas de différence significative dans l'évolution du $\mathrm{pH}$, ou de tout autre variable mesurée, pour les teneurs en $\mathrm{O}_{2}$ de 5 à $20 \%$. L'entreposage s'accompagnait d'une diminution de l'activité protéolytique spécifique en croûte. Celle-ci était toutefois plus élevée chez le témoin que chez les échantillons traités à 25 et $50 \%$ de $\mathrm{CO}_{2}$. Les témoins perdaient davantage de poids au cours de l'entreposage que les fromages traités en AC, ce qui se traduisait par une plus grande fermeté de la croûte. L'entreposage à $50 \%$ de $\mathrm{CO}_{2}$ générait, pour sa part, des croûtes moins fermes qu'à $25 \%$ de $\mathrm{CO}_{2}$. Une grande fermeté de la croûte des fromages s'accompagnait d'une grande élasticité. Les pâtes des fromages entreposés en $\mathrm{AC}$ à $50 \%$ de $\mathrm{CO}_{2}$, étaient nettement plus dures que celles à 10 ou $25 \%$, ce qui est probablement associé au $\mathrm{pH}$ plus acide de ces fromages.
\end{abstract}

\section{Fromage / fermeté / azote / dioxyde de carbone / oxygène}

\begin{abstract}
Texture and evolution of $\mathrm{pH}$ of Brie-type cheeses stored in controlled atmospheres. Brie-type cheeses were stored at $7{ }^{\circ} \mathrm{C}$ under controlled atmosphere (CA) of oxygen $(0,5,10$ or $25 \%), \mathrm{CO}_{2}(0,5,10,25$ or $50 \%)$ and nitrogen (30 to $\left.70 \%\right)$. The effect of $\mathrm{CA}$ on the evolution of $\mathrm{pH}$, proteolytic activity and texture of the cheeses was studied. A decrease of lactic acid concentration occurred under aerobic conditions, which was accompanied by a rise in $\mathrm{pH}$. There was no significant effect of $\mathrm{O}_{2}$ concentration on the evolution of $\mathrm{pH}$, in the 5 to $20 \% \mathrm{O}_{2}$ range. There was a drop in specific proteolytic activity (SPA) in the rind during storage. The SPA remained higher in control samples as compared to those under 25 or $50 \% \mathrm{CO}_{2}$. Control cheeses had higher weight losses during storage than those stored under $\mathrm{CA}$, and this was accompanied by an increased firmness of the rind. Storage in CA with $50 \% \mathrm{CO}_{2}$ generated cheese rinds having less firmness than those stored at $25 \% \mathrm{CO}_{2}$. On the other hand, curds stored at $50 \% \mathrm{CO}_{2}$ were harder that those treated with 10 or $25 \% \mathrm{CO}_{2}$, which is probably related to the lower $\mathrm{pH}$.
\end{abstract}

\section{Cheese / firmness / nitrogen / carbon dioxide / oxygen}

\footnotetext{
* Correspondance et tirés-à-part

Tél. : 450-773-1105 ; fax : 450-773-8461 ; e-mail : marcottem@em.agr.ca
} 


\section{INTRODUCTION}

Le déroulement de l'affinage du fromage de type Brie est influencé par les flores microbiennes, les enzymes ajoutées, le $\mathrm{pH}$, l'activité de l'eau ou l'humidité relative (HR) [3], la température et la composition de l'atmosphère entourant le fromage.

L'atmosphère influence l'affinage du fromage $[4,9]$ en raison de son effet sur la physiologie des microorganismes [8]. Une atmosphère modifiée au $\mathrm{NH}_{3}$ permet une élévation du $\mathrm{pH}$ et un ramollissement du fromage beaucoup plus rapide qu'en atmosphère normale $[6,10]$. D'autre part, une teneur réduite en $\mathrm{O}_{2}$ permet de ralentir le métabolisme des microorganismes aérobies, telles les moisissures, et l'ajout de gaz carbonique dans la composition du mélange de gaz a un effet bactéricide sur la croissance des microorganismes [5]. La présence de $\mathrm{CO}_{2}$ affecte la croissance de Penicillium camemberti [1], mais on ne sait toutefois pas si cette action du $\mathrm{CO}_{2}$ se traduit par des effets sur la maturation du fromage.

Le but de ce travail était de déterminer l'effet de l'atmosphère contrôlée (AC) sur l'évolution du $\mathrm{pH}$, de la flore lactique et de la texture de fromages de type Brie ainsi que sur les activités protéolytiques sous croûte.

\section{MATÉRIEL ET MÉTHODES}

\subsection{Spécifications des fromages}

Cette étude a été menée avec quatre productions industrielles de fromages de type Brie de $1,3 \mathrm{~kg}$, ayant $20 \mathrm{~cm}$ de diamètre et $3,5 \mathrm{~cm}$ de hauteur. Au moment d'être mis en atmosphère contrôlée (AC), les fromages avaient $12 \mathrm{j}$ d'affinage en usine et étaient prêts à être emballés ; J+0 correspond donc au jour de fabrication et $\mathrm{J}+12 \mathrm{au}$ moment de l'application du traitement d'AC. Les fromages $\mathrm{J}+12$ avaient en moyenne un $\mathrm{pH}$ de 5,04, 47,7 \% d'humidité, $34,7 \%$ de matières grasses et 1,3\% de sel.

\subsection{Dispositif pour la conservation des fromages sous AC}

Les fromages étaient placés dans des contenants rigides de 7,5 L équipés d'une sortie et d'une entrée facilement détachables permettant la circulation des gaz en continu. Les diverses AC et les lots de fromages sur lesquels ils ont été testés apparaissent dans le tableau I. Avant injection dans les contenants, les gaz ont été humidifiés par barbotage dans l'eau, permettant d'obtenir une humidité relative comprise entre 95 et $99 \%$. Cinq contenants servaient à étudier l'effet de chaque traitement d'AC.

Les fromages ont été conservés à $7^{\circ} \mathrm{C}$ dans les contenants hermétiques où les mélanges gazeux circulaient en continu. Le fromage témoin ( $\mathrm{T}$ ) a été laissé dans son emballage sur la tablette de la chambre froide à $7^{\circ} \mathrm{C}$.

\subsection{Analyses}

La composition des gaz prélevés dans les contenants hermétiques a été déterminée

Tableau I. Pertes de poids (en $\%$ du poids original) des fromages au cours de l'entreposage.

Table I. Weight losses (in \% of original weight) of the cheeses during storage.

\begin{tabular}{rrrrrrr}
\hline \multicolumn{3}{c}{ Traitement } & & \multicolumn{2}{c}{ Temps d'entreposage (j) } \\
\cline { 1 - 3 } \cline { 5 - 6 } $\mathrm{CO}_{2}$ & $\mathrm{O}_{2}$ & $\mathrm{~N}_{2}$ & & 47 & 69 \\
\hline 0 & $21 *$ & $79 *$ & & 5,6 & 13,4 \\
25 & 20 & 55 & & 2,4 & - \\
25 & 10 & 65 & & 2,5 & 3,2 \\
25 & 5 & 70 & & 1,9 & 2,4 \\
50 & 20 & 30 & & 1,3 & 1,7 \\
50 & 10 & 40 & & 2,5 & 1,5 \\
50 & 5 & 45 & & 2,2 & 1,6 \\
0 & 0 & 100 & & 2,2 & 1,8 \\
0 & 5 & 95 & & 1,5 & 2,8
\end{tabular}

* Témoin, dans son emballage commercial et entreposé sans atmosphère contrôlée.

* Control treatment, in its original packaging, and without controlled atmosphere storage. 
par chromatographie en phase gazeuse. Avec une seringue, on prélevait $1 \mathrm{~mL}$ de gaz via le septum, qui était injecté dans le chromatographe (Varian Inc., modèle 3400, Palo Alto, CA, USA). Ce dernier est équipé d'un détecteur TCD (Thermal Conductivity Detector) maintenu à $200^{\circ} \mathrm{C}$. L'analyse s'effectuait avec une colonne Porapack Q, 60/80 mesh pour le $\mathrm{CO}_{2}$ et une colonne Molecular Sieve 5A, $60 / 80$ mesh pour l' $\mathrm{O}_{2}$. La température de la colonne était ajustée à $100^{\circ} \mathrm{C}$ et celle de l'injecteur à $48^{\circ} \mathrm{C}$. Le débit du gaz vecteur, l'hélium, était de $30 \mathrm{~mL} \cdot \mathrm{min}^{-1}$.

Le dosage du lactate a été réalisé par chromatographie liquide haute pression. Un morceau de $1 \mathrm{~cm}$ d'épaisseur a été prélevé dans le centre du fromage. $\mathrm{Ce}$ morceau était mélangé à la phase mobile $\left(\mathrm{H}_{2} \mathrm{SO}_{4}, 0,008 \mathrm{~N}\right)$ dans un rapport phase mobile:fromage de 4:1, homogénéisé au Polytron puis centrifugé à froid 15 min à $3500 \times g$. Le surnageant ainsi obtenu était filtré sur une membrane $0,45 \mu \mathrm{m}$ (Millipore, Bedford, MA, USA) et analysé avec la méthode de Gardner et al. [2].

Pour la détermination du $\mathrm{pH}$, un morceau de pâte $(2 \mathrm{~g})$ a été ajouté à $18 \mathrm{~mL}$ d'eau distillée, broyé à l'ultraturax et le $\mathrm{pH}$ était directement mesuré dans le broyat obtenu.

Pour le dénombrement des bactéries lactiques, quelques grammes de pâte de fromage étaient dilués 1:9 dans de l'eau physiologique, puis broyés à l'ultraturax. Les dilutions appropriées dans une eau peptonée $(0,1 \%)$ stérile étaient mises en boîtes de Pétri sur milieu MRS gélosé (Difco, Detroit, MI, USA). Puisque ce fromage particulier avait été inoculé avec une culture de Streptococcus thermophilus, les colonies ont été dénombrées après une incubation des boîtes $3 \mathrm{j}$ à $43^{\circ} \mathrm{C}$.

L'activité protéolytique a été déterminée en utilisant une modification de la méthode de BCA-Pierce (Pierce Chemical Co., Rockford, IL, USA). Les fromages ont été congelés $\left(-20^{\circ} \mathrm{C}\right)$ jusqu'au moment de l'analyse. Nous avions préalablement vérifié que la congélation n'influençait pas l'activité protéolytique dans les échantillons. Afin de s'assurer que la solution de caséines ne contenait pas de peptides, une étape de purification a été réalisée comme suit: (1) dissolution de $1 \mathrm{~g}$ de caséine (caséine purifiée Sigma) dans $100 \mathrm{~mL}$ de tampon citrate $\mathrm{pH} 6$; (2) ultrafiltration de la solution de caséine sur Centriprep-10 concentrators (Amicon, seuil de coupure $=$ 10000 , centrifugation à $3000 \times g$ pendant $1 \mathrm{~h} \mathrm{30);(3)} \mathrm{rétentat} \mathrm{remis} \mathrm{à} \mathrm{volume} \mathrm{avec} \mathrm{du}$ tampon citrate $100 \mathrm{mmol} \cdot \mathrm{L}^{-1}, \mathrm{pH}$ 6. À la sortie du congélateur, la croûte des fromages était découpée à l'aide d'un fil à beurre en prenant bien soin de ne pas entraîner de pâte. Environ 1,5 g de croûte était dilué au 1/3 dans du tampon citrate, puis broyé au polytron jusqu'à l'obtention d'une pâte homogène. Ensuite, 1,5 mL de chaque homogénat était centrifugé $10 \mathrm{~min}$ à $12000 \times g$ dans des tubes Eppendorf. Le surnageant $\mathrm{S} 1$ a été récupéré dans un tube Eppendorf de $1,5 \mathrm{~mL}$ et servait aux dosages de la teneur en protéines et à la détermination de l'activité protéolytique.

La teneur en protéine (TP) des échantillons a été déterminée comme suit : (1) dilution du surnageant $\mathrm{S} 1$ au $10^{\mathrm{e}}$ dans du tampon citrate $100 \mathrm{mmol} \cdot \mathrm{L}^{-1}, \mathrm{pH} 6$; (2) après agitation, $10 \mu \mathrm{L}$ de chaque échantillon dilué, ou de tampon dans le cas du blanc, injectés dans un des puits de la microplaque ; (3) $200 \mu \mathrm{L}$ de réactif $\mathrm{BCA}$ ajoutés dans chaque puits ; (4) microplaques placées dans un incubateur à $37^{\circ} \mathrm{C}$ pendant $30 \mathrm{~min}$; (5) lecture de l'absorbance des échantillons dans les microplaques à $570 \mathrm{~nm}$ (Perkin Elmer - Lamda Reader). La concentration en protéines était définie en se rapportant à une courbe étalon établie dans les mêmes conditions avec une solution de «bovine serum albumin» (BSA).

L'activité protéolytique (AP) était dosée de la façon suivante: (1) dans un tube épendorf $(1,5 \mathrm{~mL})$, on prélève $100 \mu \mathrm{L}$ de surnageant (S1) ; (2) ajout de $400 \mu \mathrm{L}$ de solution de caséine (rétentat UF) ; (3) incubation des préparations $3 \mathrm{~h}$ à 
$37{ }^{\circ} \mathrm{C}$ dans un bloc chauffant ; (4) réaction stoppée par ajout dans les tubes de $60 \mu \mathrm{L}$ d'acide trichloroacétique $100 \%$ (poids/ vol) ; (5) centrifugation des tubes 10 min à $12000 \times g$; (6) $10 \mu \mathrm{L}$ du nouveau surnageant S2 prélevés dans les puits des microplaques ; (7) $200 \mu \mathrm{L}$ de réactif BCA rajoutés dans chaque puits ; (8) microplaques placées dans un incubateur à $37^{\circ} \mathrm{C}$ pendant $30 \mathrm{~min}$; (9) l'absorbance lue à $570 \mathrm{~nm}$. La concentration en petits peptides libérés est définie en se rapportant à une courbe étalon établie dans les mêmes conditions avec une solution de BSA. Puisque l'échantillon de fromage contenant les protéases pouvait également apporter des peptides, la TP a servi à tenir compte de cette contribution à l'AP et a été utilisée dans la détermination de l'activité protéolytique spécifique en croûte (APSC). On estime donc l'APSC en employant la formule suivante :

$$
\begin{aligned}
\mathrm{APSC}= & ((\mathrm{AP} 1 / \mathrm{TP} 1)+(\mathrm{AP} 2 / \mathrm{TP} 2) \\
& +(\mathrm{AP} 3 / \mathrm{TP} 3)) / 3
\end{aligned}
$$

où 1,2 et 3 sont les réplicats.

Les fromages ont été analysés avec un texturomètre (modèle 4201, Instron Inc., Canton, MA, USA) équipé d'une cellule de 50 N. Le logiciel de Instron Series XII a été utilisé pour l'acquisition de données et de contrôle durant les tests. À chaque prélèvement, deux séries de tests ont été réalisées : pénétrométrie et compression (TPA). Le test de pénétrométrie est utilisé pour étudier les propriétés rhéologiques de la croûte des fromages, le test de compression pour étudier les propriétés de la pâte. Le test de pénétrométrie, destiné à évaluer la fermeté de la croûte (force requise pour la percer), était réalisé dans les conditions suivantes : (1) vitesse du poinçon : $20 \mathrm{~mm} \cdot \mathrm{min}^{-1}$; (2) $60 \%$ déformation = $60 \%$ de la hauteur de l'échantillon; (3) diamètre du poinçon : $6,5 \mathrm{~mm}$. Suivant les emplacements d'un gabarit en plexiglass spécialement conçu à la taille des fromages, 3 échantillons sont prélevés à $2 \mathrm{~cm}$ du bord, et 3 autres au centre des fromages. Ainsi les échantillons sont toujours prélevés de la même façon d'un fromage à l'autre. Le test de compression de la pâte, qui détermine la force requise pour atteindre un niveau précis de déformation, était réalisé dans les conditions suivantes: (1) vitesse de compression: $20 \mathrm{~mm} \cdot \mathrm{min}^{-1}$, et $60 \%$ déformation ; (2) diamètre du plateau de compression : $35 \mathrm{~mm}$. Suivant les emplacements du gabarit, 5 carottes sont prélevées à l'emporte-pièce $($ diamètre $=25 \mathrm{~mm})$ à $2 \mathrm{~cm}$ du bord, et 2 autres dans le centre du fromage. De chaque côté de la carotte, la croûte est enlevée au moyen d'un fil à beurre, afin d'obtenir une hauteur standard de $30 \mathrm{~mm}$. On a mesuré la force nécessaire pour atteindre une déformation de $60 \%$. Les échantillon ont été conservés à $7{ }^{\circ} \mathrm{C}$ jusqu'au moment de l'analyse.

Au moins deux essais ont été effectués pour chaque traitement. Pour chaque essai, deux fromages subissaient chaque traitement, et les analyses étaient effectuées sur chacun de ceux-ci. Les analyses statistiques ont été réalisées avec le logiciel InStat (GraphPad, San Diego, USA). Les données (pH, APSC, comptes viables) présentaient une variabilité considérable à l'intérieur d'une même répétition, avec des coefficients de variation typiquement à $20 \%$.

\section{RÉSULTATS ET DISCUSSION}

\subsection{Effet de l'AC sur l'évolution du pH}

Les moisissures consomment l'acide lactique lors de leur croissance en conditions aérobies. Ceci entraîne une hausse du $\mathrm{pH}$. Une corrélation $\left(\mathrm{R}^{2}=0,88\right)$ a d'ailleurs été trouvée entre le $\mathrm{pH}$ et la teneur en acide lactique, et ce, pour les traitements ayant de $0-25 \%$ de $\mathrm{CO}_{2}$. Cette régression nous permet de voir que l'assimilation complète du lactate a lieu lorsque le $\mathrm{pH}$ du fromage atteint environ 6,8. Audelà de $\mathrm{pH} 6,8$ on peut penser que les moisissures se servent des protéines comme 


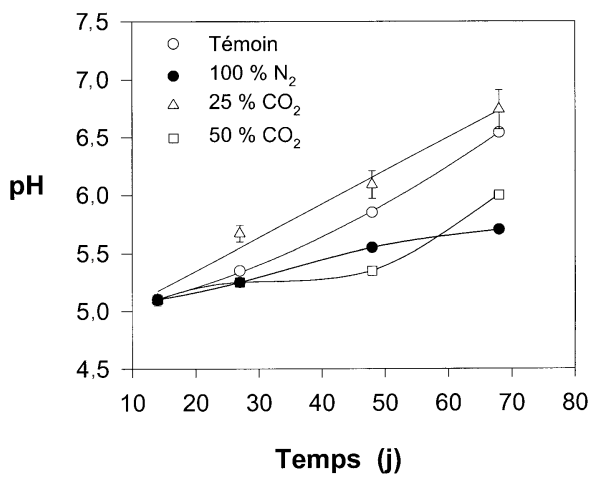

Figure 1. Effet de l'entreposage sous atmosphère contrôlée sur l'évolution du pH des fromages. Les barres d'erreur constituent l'erreur standard sur la moyenne et, pour raison de clarté, sont placées sur une seule courbe.

Figure 1. Effect of storage under controlled atmosphere on the evolution of $\mathrm{pH}$ in cheeses. Error bars represent standard error on means, and are placed on only one curve for reasons of clarity.

source de carbone, et une production plus importante d'ammoniaque est à prévoir.

L'entreposage en anaérobiose affecte l'évolution du $\mathrm{pH}\left(100 \% \mathrm{~N}_{2}\right.$, Fig. 1). L'utilisation de l'acide lactique est plus importante en conditions aérobies, ce qui entraîne une plus forte montée du $\mathrm{pH}$ en présence d'oxygène.

Plusieurs traitements AC comprenaient des conditions d' $\mathrm{O}_{2}$ de 5,10 ou $20 \%$ (Tab. I). Aucune différence significative dans l'évolution du $\mathrm{pH}$, ou de tout autre paramètre, n'a été observée pour les teneurs en $\mathrm{O}_{2}$ de 5 à $20 \%$. En conséquence, comme les effets de l'oxygène étaient moins marqués que ceux de la teneur en $\mathrm{CO}_{2}$, ce dernier aspect a fait l'objet d'un examen plus poussé.

La présence de $\mathrm{CO}_{2}$ affectait l'évolution du $\mathrm{pH}$, mais seulement à une très forte teneur $(50 \%$, Fig. 1$)$. Il a été démontré que des teneurs en $\mathrm{CO}_{2}$ de $30 \%$ dans l'atmosphère ralentissent la croissance de Penicillium camemberti [1]. Nos résultats vont dans ce

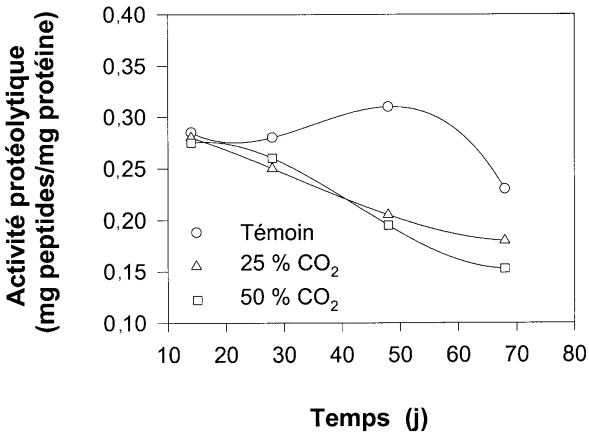

Figure 2. Effet de l'entreposage sous atmosphère contrôlée sur l'évolution de l'activité protéolytique spécifique sous croûte des fromages.

Figure 2. Effect of storage under controlled atmosphere on the evolution of the specific proteolytic activity of the cheeses.

sens, puisque la désacidification n'est affectée qu'à $50 \%$ de $\mathrm{CO}_{2}$. Toutefois, le $\mathrm{CO}_{2}$ en soi peut acidifier le milieu, et il nous fallait donc réaliser les analyses HPLC et protéolytiques pour déterminer dans quelle mesure les différences dans les courbes de $\mathrm{pH}$ étaient associées au $\mathrm{CO}_{2}$ ou à une modification des activités biochimiques du Penicillium.

\subsection{Effet de l'AC sur l'évolution de l'activité protéolytique}

L'entreposage s'accompagnait d'une diminution de l'activité protéolytique spécifique en croûte (APSC) (Fig. 2). L'analyse statistique révèle que l'APSC à J+48 est significativement plus élevée chez le témoin que chez les échantillons traités à 25 et $50 \%$ de $\mathrm{CO}_{2}$. Ainsi, le traitement en $\mathrm{AC}$ avec teneurs élevées en $\mathrm{CO}_{2}$ a diminué l'APSC, et diverses hypothèses peuvent être émises pour expliquer ce phénomène : (1) biomasse réduite en $\mathrm{AC}$; (2) mortalité fongique accélérée en $\mathrm{AC}$; (3) inhibition de la synthèse des protéases en $\mathrm{AC}$; (4) instabilité des protéases en $\mathrm{AC}$; (5) $\mathrm{pH}$ plus bas. 


\subsection{Effet de l'AC sur l'évolution de la flore bactérienne dans les fromages}

La population lactique des caillés témoins demeurait stable entre $\mathrm{J}+27$ et $\mathrm{J}+48$ d'entreposage à $7 \times 10^{8} \mathrm{UFC} \cdot \mathrm{mL}^{-1}$. Toutefois, une forte diminution se notait par la suite, puisque celle-ci n'était plus que de $6 \times 10^{7} \mathrm{UFC} \cdot \mathrm{mL}^{-1}$ à $\mathrm{J}+69$. Les fromages traités à $5-25 \%$ de $\mathrm{CO}_{2}$ présentaient des courbes de mortalité semblables. Une mortalité accrue semblait se noter chez les fromages en AC de $50 \%$ de $\mathrm{CO}_{2}$, mais les fortes variations observées ne permettaient pas de conclure.

\subsection{Effet de l'AC sur l'évolution des poids des fromages}

Les profils de pertes de poids sont présentés dans le tableau I. Les pertes en poids étaient importantes chez le témoin, mais réduites au cours de l'entreposage en $\mathrm{AC}$ en raison de l'humidification du gaz d'AC. Les résultats suggèrent que des pertes (à $\mathrm{J}+48$ ) légèrement inférieures (jusqu'à $1 \%$ ) se produisent lorsque la teneur en $\mathrm{CO}_{2}$ est de $50 \%$. Ceci pourrait être associé à une assimilation réduite du lactate et des autres constituants du fromage, ce qui résulterait en une production inférieure de $\mathrm{CO}_{2}$ métabolique. Il est également possible qu'une fraction de la perte en eau observée sans $\mathrm{AC}$ soit associée à la cristallisation du phosphate de calcium.

\subsection{Effet de l'AC sur les propriétés physiques des fromages}

Il était plus difficile de provoquer une rupture de la croûte dans les échantillons témoins (Fig. 3) que dans ceux qui étaient en AC. Les témoins perdaient davantage de poids que les traitements en AC (Tab. I) et il est possible que cette fermeté accrue de la croûte soit due tout simplement à son assèchement. Une cristallisation du phosphate de calcium pourrait également y contribuer. L'entreposage à $50 \%$ de $\mathrm{CO}_{2}$

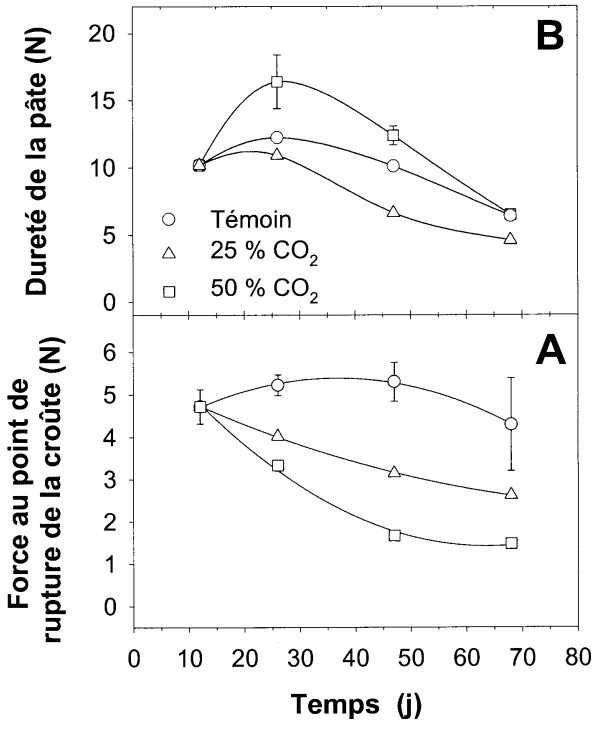

Figure 3. Effet de l'entreposage sous atmosphère contrôlée sur $(\mathrm{A})$ la fermeté de la croûte, telle que déterminée par la force requise pour la percer et (B) la dureté de la pâte, telle que déterminée par la force requise pour atteindre $60 \%$ de déformation. Les barres d'erreur constituent l'erreur standard sur la moyenne et, pour raison de clarté, sont placées sur une seule courbe.

Figure 3. Effect of storage under controlled atmosphere on (A) the firmness of the rind, as determined by the force required for its rupture and (B) the hardness of the curd, as determined by the force required to obtain a 60\% deformation. Error bars represent standard error on means, and are placed on only one curve for reasons of clarity.

donnait, pour sa part, des croûtes moins fermes que celles des fromages entreposés à $25 \%$ (Fig. 3). La fermeté moins élevée des croûtes en AC de $50 \%$ (Fig. 3) s'accompagnait d'une rupture rapide de celles-ci. Ainsi, une plus grande fermeté de la croûte des fromages était associée à une plus grande élasticité. En effet, la rupture de la croûte des fromages témoins se faisait lorsque le poinçon avait parcouru une distance plus grande que chez les fromages en AC de $50 \%$. 
Les traitements AC influencent également la dureté de la pâte. Les pâtes des fromages entreposés en $\mathrm{AC}$ à $50 \%$ de $\mathrm{CO}_{2}$, sont nettement plus dures que celles à 10 ou $25 \%$ (Fig. 3). Ceci est probablement associé au pH plus acide de ces fromages. En effet, l'entreposage à $50 \%$ de $\mathrm{CO}_{2}$ ralentit la désacidification du fromage (Fig. 1) et les pâtes plus acides sont plus dures. Cette observation pourrait aussi être reliée à une APSC plus faible dans les fromages entreposés avec $50 \%$ de $\mathrm{CO}_{2}$. Les pâtes des fromages témoins ont une dureté supérieure à celles des fromages en $\mathrm{AC}$ de $25 \%$ (Fig. 3) malgré des $\mathrm{pH}$ similaires (Fig. 2). On peut à nouveau se demander si l'assèchement accru des fromages témoins explique ce phénomène.

Murr et Morris [7] ont observé qu'une concentration en $\mathrm{CO}_{2}$ inférieure à $5 \%$ inhibait de façon significative la croissance de champignons et des concentrations supérieures à 25 ou $50 \%$ les endommageaient sérieusement. Nos résultats s'inscrivent dans cette ligne. Les fromages exposés à $25 \%$ de $\mathrm{CO}_{2}$ montrent de légères modifications, mais les changements physico-chimiques deviennent notables à $50 \%$ de $\mathrm{CO}_{2}$. L'entreposage en AC permet de réduire la déshydratation et de diminuer la vitesse de remontée du $\mathrm{pH}$, ce qui laisse entrevoir la possibilité d'augmenter la durée d'entreposage. Toutefois, ceci s'acompagne d'une modification de la texture des fromages, et il reste à évaluer les conséquences de l'AC sur la saveur des fromages.

\section{REMERCIEMENTS}

Nous remercions N. Gardner, J. Fortin et N. Robert pour leur aide technique et scientifique, ainsi que M. Guillet pour le support statistique, J. Picque dans la planification expérimentale et L. Charlebois pour le traitement de texte.

\section{RÉFÉRENCES}

[1] Doyon G., Gagnon J., Lafontaine D., Désilets C., Champagne C.P., The effect of carbon dioxide on the growth of Penicillium camemberti, Microbiol. Aliments Nutr. 15 (1997) 291-297.

[2] Gardner N.J., Savard T., Obermeier P., Caldwell G., Champagne C.P., Selection and characterization of mixed starter cultures for lactic fermentation of carrot, cabbage, beet and onion vegetable mixtures, Int. J. Food Microbiol. 64 (2001) 261-275.

[3] Kiermeier F., Wolfseder H., Behaviour of packaged cheese. II. Effect of packaging on weight losses of Camembert cheese, Lebensm. Unters. Forsch. 149 (1972) 218-223.

[4] Kiermeier F., Wolfseder H., Behaviour of packaged cheese. III. Effect of packaging on gas-metabolism of Camembert, Lebensm. Unters. Forsch. 150 (1972) 75-83.

[5] Kramarczyk K., The effects of MAP on the growth and spore germination of an Alternaria species and a Botrytis species isolated from blueberries, 1978, M.S. Thesis, Rutgers Univ., New Brunswick, NJ, USA, $37 \mathrm{p}$.

[6] Le Graet Y., Lepienne A., Brulé G., Ducruet P., Migration of calcium and inorganic phosphate in Camembert-type cheeses during ripening, Lait 63 (1983) 317-332.

[7] Murr D.P., Morris L.L., Effect of storage atmosphere on postharvest growth of mushrooms, J. Amer. Soc. Hortic. Sci. 100 (1975) 298-301.

[8] Stehle G., Packaging of soft cheeses, NeueVerpackung 39 (1986) 146-155.

[9] Sweetman A., Active wrapping for soft cheese, Dairy Ind. Int. 56 (1991) 28-29.

[10] Vassal L., Monnet V., Le Bars D., Roux C., Gripon J.C., Relation entre le pH, la composition chimique et la texture des fromages de type Camembert, Lait 66 (1986) 341-351. 